\title{
License-aware Service Selection
}

\author{
G.R. Gangadharan ${ }^{\dagger}$, Marco Comerio $^{\ddagger}$, Hong-Linh Truong ${ }^{\S}$, \\ Vincenzo D’Andrea ${ }^{\dagger}$, Flavio De Paoli ${ }^{\ddagger}$, Schahram Dustdar $^{\S}$ \\ $\dagger$ University of Trento, Trento, Italy \\ \{gr, dandrea\}@disi.unitn.it \\ $¥$ University of Milano - Bicocca, Milano, Italy \\ \{comerio,depaoli\}@disco.unimib.it \\ $\S$ Vienna University of Technology, Austria \\ \{truong, dustdar\}@infosys.tuwien.ac.at
}

\begin{abstract}
The distribution of services spanning across organizational boundaries raises problems related to intellectual value that are less explored in current service oriented research. Being a way to manage the intellectual rights between service consumers and service providers, licenses are critical to be considered in services. A service license describes the terms and conditions for the use and access of the service in a machine interpretable way. The proliferation of services that offer similar functionalities requires the selection process to be enhanced together with consideration of licenses. In this paper, we analyze current service licensing practices and present a novel approach for service selection process based on matching the offered licensing specifications (primarily dealing with scope of rights and financial terms) by providers with the requested licensing specifications by consumers.
\end{abstract}

\section{Introduction}

Given that services are changing the way organizations build, deploy, and manage their information technology assets, it makes sense to rethink rights offered to these organizations in connection with the use of services. As the nature of services differs significantly from traditional software and components and services are being accessed and consumed in a number of ways, services prevent the direct adoption of classical software and component licenses [6]. A service license includes all transactions between the licensor and the licensee, in which the licensor agrees to grant the licensee the right to use and access the service under predefined terms and conditions.
In general, a service provider defines individual services with corresponding service licenses (offered license specifications) which consumers should follow. Often, service consumers are interested in selecting a service based on certain licensing clauses (requested license specifications). For a set of requested licensing clauses, there can be several licenses that differ in the set of offered license specifications. While many researches describe the selection of services based on functional and non-functional properties, the issue of matching service requests to offers has not yet been extensively analyzed considering the intellectual rights associated to services. This paper proposes an approach for enhancing service selection with a novel process based on matching the offered licensing specifications with the requested licensing specifications.

The rest of paper is organized as follows. Section 2 analyzes the rights associated with services and investigates the pricing mechanisms for services. Section 3 briefly presents the concepts of service license specification. The process of license aware service selection (involving the clauses of scope of rights and financial terms) is elucidated in Section 4. Section 5 discusses related work followed by concluding remarks in Section 6.

\section{Analyzing Current Service License Prac- tices}

Most software licenses [18] restrict the scope of grant by offering the rights to use object code and limited rights to make archival copy. Free/Open Source Software (FOSS) [13] licenses allow free access to the source code of software. In contrast, the nature of services induces broader license grants to support reusability of services with other applications. Most services may even require the rights of 
modifications to provide value added services. A licensee may not show interest if a service prohibits value addition with other services or applications. As the scope of rights is the provision stipulating the rights the licensor grants to the licensee, the following scenarios arise in services that determine the flexibility of rights.

As service interfaces together with bindings are publicly available, several services could be created with the same interface with or without modifications. These services can vary in their performances. Following are common scenarios of reproducing interface with modifications.

- The interface of a service could be modified by changing the name of some operations such as for translation (the expression of a service in a language other than that of the original version).

- The interface of a service could be reproduced by data translation.

- The interface of a service could be modified by making changes in service parameters or by some preprocessing and/or post-processing of the given service.

In a more exclusive manner, a WSDL file representing the interface and its implementation representing the behavior could be licensed differently. The WSDL file could even be licensed with a clause denying modification of the service interface, though the interface source code is openly available. Thus, irrespective of the openness of service implementation, the restrictions on scope of rights regarding the modification of WSDL file are highly significant in services.

In line with software, a service provider could distribute (possibly with a fee) a service software to other providers. Another provider will then be able to offer a similar service to the original one, possibly with different data or using a different business model.

A service could allow its implementation to be used as an executable by other services. In contrast to this model, a service even could allow other services to modify its implementation.

Though there are examples of service licenses in practical use (by Amazon, Google, Yahoo!), to the best of our knowledge, there appears to be no conceptualization of service licensing in general. The business and legal contractual information are not described at a detailed level by the services research community, either in industry or academia.

A service consumer assesses how much he/she can afford to pay for the service that will add value to him/her and how will the service be consumed. From the perspective of service providers, the estimation of cost of producing a service and amount of return on investment plays the critical role in determining the price.
Consider that a Stockquote service is provided by a service provider. The value of this service lies in the on-time delivery of stock prices to consumers. This type of service is based on critical data that change continuously and requires a considerable investment in provision of the service.

Certain consumers may expect a Stockquote service to deliver stock quotes in real time without delay for their applications. In this case, a provider can offer the service based on varying levels of functionalities and nonfunctional properties, as demanded by consumers. Thus, the price of a service becomes directly proportional to the features offered.

In both cases, service consumers define in what way the service is going to be used by them. A service consumer may invoke the service unlimited times. In another case, consumers may wish to pay based on the number of times they use a given service. We can generalize the models for pricing of services as follows [9].

- Transaction based model allows service providers to charge for each use, as the license defines the term 'use'. The use of services can be continuously recorded and monitored by service management systems. This model of pricing is quite similar to charging true utilities like electricity and water.

- Subscription based model allows service consumers to purchase services for a fixed term, during which time they automatically receive full support from service providers including any upgrades or feature enhancements. Service consumers typically pay periodically. The subscription model is based on the premise that both service providers and service consumers benefit from automatic continuous updating.

\section{Service License Specification}

In the business domain, consumer confidence is established through a contract with the service provider. In SOC, service level agreements (SLA) and policies support these contractual terms. A service license primarily focuses on the usage and provisioning terms of services. A service license may include the SLA terms. Thus, a service license is broader than the scope of SLA, protecting the rights of service providers and service consumers. In general, an agreement is negotiated between the service provider and the service consumer and agree upon a SLA that covers a service (or a group of services). The agreement is terminated when either of the party terminates or violates the agreement. If one of the partners violates the agreement, the agreement might be renegotiated (in case of recoverable violation). In case of a service license, there is a service provider that plays the main role of the licensor. There could be many 
service consumers (the licensees) binded by the service license. However, a license seems as if the licenses were not even involved in the transactions between the licensor and the licensees.

In general, a service license is used to achieve the following objectives.

1. Subject: Describing the information regarding the service being licensed and other related information such as an unique identification code, the details of the service provider, and so on.

2. Scope of Rights: Defining the extent to which the service could be used, accessed, and value added, on the basis that any use outside the scope of license would constitute an infringement.

3. Financial Terms: Explaining payment and charging terms.

4. Warranties, Indemnities, and Limitation of Liability: Specifying delivery terms (regarding quality of services and performance), acceptance terms, warranties, and limiting the liability of providers in case of failures.

5. Evolution: Declaring the rights over future versions and over evolved services.

Current SLA and policies specifications for services (WSLA [12], SLANG [14], WSOL [15], WS-Policy [16]) define what to measure/monitor and describe payments/penalties. Generally, all the specifications focus on the QoS and the terms and conditions agreed by a provider and a consumer. License clauses are unexplored by current service description standards and languages (as mentioned above). We have proposed a language ODRL-S [7] by extending the Open Digital Rights Language (ODRL) [10] to implement the clauses of service licensing.

A ODRL-S based license can be linked to service description and other information, e.g., using a Web services catalog based on XML. Applications that need to discover resources and services can use a service that offers this catalog information via Web or Web services protocols. Viewing a service license as a resource, this provides a foundation for an interoperable mechanism for license-aware service selection and management of services.

\section{License-aware Selection Process}

Generally, a service consumer looks up a service management system for services with a specific functionality. The service selection process tries to find if any service advertisements given by providers match the request of consumers. There may be always the possibility of more than one services, offering similar functionality that differ in their licenses.

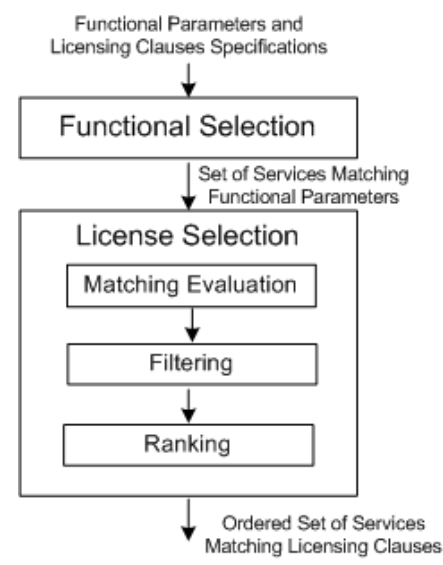

\section{Figure 1. License-aware service selection process}

As shown in Figure 1, we propose to extend the traditional functionality-based service selection process with an additional selection based on licenses.

The Functional Selection has the goal of finding a set of services that totally satisfy the functionalities required by a consumer. This activity finds a set of candidate services; each one providing the same functionality but associated with different licenses. Although functionality-based service selection is combined with our license-aware selection, it is out of the scope of this paper.

The License Selection includes three phases: Matching Evaluation, Filtering and Ranking. The Matching Evaluation computes two different indicators (Degree and Distance) expressing the matching degree between a license clause required by a consumer and a respective license clause offered by a provider. The Degree shows if an offer matches a request and is expressed by a value in the range [0..1], where 0 means 'no match' and 1 means 'exact match'. The Distance indicator is introduced to capture additional information about the matching. In the case of 'exact match', we evaluate how much the offer dominates the request (Distance $\geq 0$ ) and in the case of 'no match', we evaluate how much the offer is far from satisfying the request (Distance $\leq 0)$.

Evaluating the values for license specifications involves the following kinds of data.

- Scope of Rights are expressed as distinct values (for example, adaptation, composition, and derivation).

- Financial Terms are expressed in a range of values (can be any value between a minimum value and a maximum value). 


\begin{tabular}{|l|l|l|l|}
\hline Request & Offer & Request versus Offer & Redefinition \\
\hline Composition & Adaptation & Composition $\supset$ Adaptation & Composition \\
\hline Derivation & Adaptation & Derivation $\supset$ Adaptation & Derivation \\
\hline Derivation & Composition & Derivation $\supset$ Composition & Derivation \\
\hline Adaptation & Composition & Adaptation $\subset$ Composition & Composition \\
\hline Adaptation & Derivation & Derivation $\subset$ Adaptation & Derivation \\
\hline Composition & Derivation & Derivation $\subset$ Composition & Derivation \\
\hline
\end{tabular}

Table 1. Subsumption rules over Scope of Rights elements

\begin{tabular}{|l|l|l|l|}
\hline Request & Offer & Degree & Distance \\
\hline Derivation & Composition & 0 & -0.5 \\
\hline Derivation & Adaptation & 0 & -1 \\
\hline Composition & Adaptation & 0 & -0.5 \\
\hline Composition & Derivation & 1 & +0.5 \\
\hline Adaptation & Composition & 1 & +0.5 \\
\hline Adaptation & Derivation & 1 & +1 \\
\hline
\end{tabular}

Table 2. Indicators in case of subsumption over Scope of Rights elements

\begin{tabular}{|c|c|c|}
\hline Condition & Degree & Distance \\
\hline $\operatorname{minOffV}<\operatorname{minReq} V$ & $1-\frac{\min R e q V-\min O f f V}{\max O f f V-\min O f f V}$ & 0 \\
\hline $\max O f f V>\max R e q V$ & $1-\frac{\max O f f V-\max \operatorname{Req} V}{\max O f f V-\min O f f V}$ & 0 \\
\hline $\begin{array}{l}\operatorname{minOffV}<\operatorname{minReqV} \text { and } \\
\operatorname{maxOffV}>\operatorname{maxReqV}\end{array}$ & $1-\frac{\operatorname{minReq} V-\min O f f V+\max O f f V-\max R e q V}{\max O f f V-\min O f f V}$ & 0 \\
\hline $\operatorname{minOffV}>\operatorname{maxReqV}$ & 0 & $-\frac{A v g(\min O f f V, \max O f f V)}{\max R e q V}$ \\
\hline maxOffV $<$ minReqV & 0 & $-\frac{\operatorname{minReqV}}{\operatorname{Avg}(\min O f f V, \max O f f V)}$ \\
\hline elsewhere & 1 & 0 \\
\hline
\end{tabular}

Table 3. Degree and Distance formulas for Financial Terms

For the clauses of Scope of Rights, if a requested license specification clause matches exactly with an offered one, the Degree is set to 1 and the Distance is set to 0 . If there is not an exact match, we can exploit the (semantic) relation of subsumption to identify compatible licences.

Assume that we have two services with different license elements say, composition and derivation. If a consumer is looking for a service allowing composition, a service license allowing derivation can also be used, because derivation subsumes composition. A consumer desiring composition cannot obviously use an offered license with adaptation. Subsumption implies a match that should occur, if the given license element is more permissive (accepts more) than the corresponding element in the other license. The subsumption rules for Scope of Rights are given in Table 1.

Table 2 lists evaluation of Degree and Distance indicators for Scope of Rights based on subsumption rules.

For what regards Financial Terms, the matching degree between the interval of requested values and the interval of offered values is evaluated using the list of formulas reported in Table 3.
The results of the Matching Evaluation are processed during the Filtering phase. A service whose license has a clause with Degree $=0$ is discarded and no longer considered in the selection process. Thus, the number of candidate services in the set of functionally matched services can be reduced. If all services are discarded (no services are able to satisfy the requested license specifications), a service with a license closer to a consumer's requested specifications will be recommended. The service recommended to a consumer is selected based on the Distance indicator. The consumer can accept or deny the recommended service (as not exactly satisfying the requested license specifications).

After the discarding of unsuitable services, the Filtering phase proceeds computing Net Degree $(\Psi)$ and Net Distance $(\Delta)$ for each license. Net Degree $(\Psi)$ provides information about how much an offered license matches a requested license. It is the sum of matching degrees of clauses of a license, represented as,

$$
\Psi=\sum_{i=1}^{n} \text { Degree }_{i}
$$


Net Distance $(\Delta)$ is a value that provides additional information about how the required clauses are matched. It is the sum of the normalized distance (n_distance) evaluated for the clauses of a license.

$$
\Delta=\sum_{i=1}^{n} n_{-} \text {distance }_{i}
$$

The value of $n_{-}$distance $_{i}$ is computed by

$$
\begin{array}{r}
n_{\text {distance }_{i}}=\frac{\text { Distance }_{i}}{\max (\mid \text { Distance })} \\
n_{-} \text {distance }_{i} \in[-1 . .1]
\end{array}
$$

where Distance $_{i}$ is the distance indicator evaluated between the required clause and the clause offered by the ith license and $\max (\mid$ Distance $\mid)$ is the maximum absolute value of distance indicator evaluated for the same requested clause among all the licenses.

The License Selection ends with the Ranking phase where the remaining candidate services are sorted according to their $\Psi$ values. If two or more services have equal $\Psi$ value, their $\Delta$ values are considered for ranking.

We explain our approach through the following example. Assume that a consumer requests for a service with the following licensing clause specifications.

1. The service should allow composition. (lines 2-4)

2. The cost of a service should not be greater than 1.50 Euros per use. (lines 5-12)

A fragment of service license describing the requested clauses (expressed in ODRL-S) is the following.

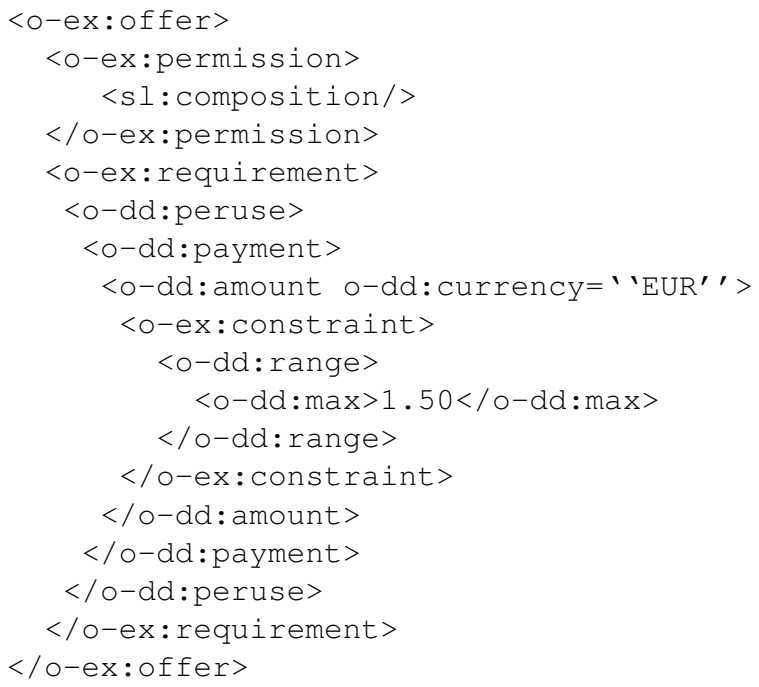

There are three services (namely $S_{1}, S_{2}$, and $S_{3}$ ) with different license specifications (see Table 4). These licenses are expressed in ODRL-S, similar to the service license previously shown, but differ in licensing clauses. The Degree and Distance indicators are evaluated as shown in Table 5.

\begin{tabular}{|l|l|l|l|}
\hline $\begin{array}{l}\text { License } \\
\text { Clauses }\end{array}$ & $L\left(S_{1}\right)$ & $L\left(S_{2}\right)$ & $L\left(S_{3}\right)$ \\
\hline $\begin{array}{l}\text { Scope of } \\
\text { Rights (SR) }\end{array}$ & Composition & Derivation & Composition \\
\hline $\begin{array}{l}\text { Cost per } \\
\text { use (Euros) }\end{array}$ & $\begin{array}{l}\min : 2.00 \\
\max : 3.00\end{array}$ & $\begin{array}{l}\min : 0.00 \\
\max : 1.00\end{array}$ & $\begin{array}{l}\min : 0.00 \\
\max : 0.50\end{array}$ \\
\hline
\end{tabular}

Table 4. Offered license specifications for services $S_{1}, S_{2}$, and $S_{3}$

\begin{tabular}{|l|l|l|l|l|}
\hline Service & $\begin{array}{l}\text { SR- } \\
\text { Degree }\end{array}$ & $\begin{array}{l}\text { Cost- } \\
\text { Degree }\end{array}$ & $\begin{array}{l}\text { SR- } \\
\text { Distance }\end{array}$ & $\begin{array}{l}\text { Cost- } \\
\text { Distance }\end{array}$ \\
\hline$S_{1}$ & 1 & 0 & $0(0)$ & $-1.6(-0.26)$ \\
\hline$S_{2}$ & 1 & 1 & $+0.5(1)$ & $3(0.5)$ \\
\hline$S_{3}$ & 1 & 1 & $0(0)$ & $6(1)$ \\
\hline
\end{tabular}

Table 5. Degree, Distance and (n-Distance) Evaluation

During the Filtering phase, $S_{1}$ is discarded as it fails to meet the requested clause specifications. The net indicators are reported in Table 6. Based on the Net Indicators, the ranking phase selects $S_{2}$ as the service that best meets the requested license specifications.

\begin{tabular}{|l|l|l|}
\hline Service & $\Psi$ & $\Delta$ \\
\hline$S_{2}$ & 2.0 & 1.5 \\
\hline$S_{3}$ & 2.0 & 1.0 \\
\hline
\end{tabular}

Table 6. Net Indicators Evaluation

\section{Related Work and Discussions}

Service selection involves matching a service description of a consumer to service descriptions of providers, with the objective of finding the most appropriate ones [8]. Many researches focus on finding of services based on functionality specifications [2]. The selection of services based on nonfunctional specifications has also been studied intensively by the research community $[17,11,1]$.

In [4], we have presented a semantic approach for selection of services by evaluating both qualitative and quantitative non-functional properties (NFPs). The approach is based on matching rules to compute numeric matching indicators. These rules are based on the ontological description of objects representing non-functional properties that are required and offered. The result is an automatic selection process that evaluates offered NFPs with respect to a set of user requests. In this paper, we have proposed a novel approach for the selection of services whose descriptions are not semantic (as in the case of ODRL-S descriptions). 
To the best of our knowledge, there is no work on selection of services based on their license specifications. Google $^{1}$ and Yahoo! ${ }^{2}$ search engines provide advanced options to retrieve contents based on requested licenses. However, these options restrict consumers with limited specifications of licenses.

The economic aspects of an utility computing service are analyzed and modeled by [5]. Interactive pricing mechanisms are defined by [3] as the subset of dynamic pricing mechanisms in a service oriented architecture. In our paper, we have proposed a way of service selection considering the price of a service as expressed by consumers.

\section{Concluding Remarks}

As a way of managing the rights between service consumers and service providers, licenses are of critical importance to services. Making the selection of services integrated with licenses is an enhanced dimension in service discovery. In this paper, we have illustrated a novel mechanism for selection of services by a match making method between license clauses requested by a consumer and offered by several providers.

We are currently extending the present service selection process to include more complex licensing clauses including warranties (can include non-functional properties) and limitation of liability. In our future work, we are planning to propose a framework that integrates the license-aware selection process with the selection of services based on matching of functional properties. We are also evaluating our approach in real industrial environments.

\section{References}

[1] D. Ardagna and B. Pernici. Adaptive Service Composition in Flexible Processes. IEEE Transactions on Software Engineering, 33(6), 2007.

[2] L. Aversano, G. Canfora, and A. Ciampi. An Algorithm for Web service Discovery through their Composition. In Proceedings of the IEEE International Conference on Web Services (ICWS), 2004.

[3] M. Bernhardt and O. Hinz. Creating Value with Interactive Pricing Mechanisms A Web Service-Oriented Architecture. In Proceedings of the International Conference on E-Commerce Technology (CEC05), 2005.

[4] M. Comerio, F. De Paoli, A. Maurino, and M. Palmonari. NFP-aware Semantic Web Services Selection. In Proceedings of the 11th IEEE International Enterprise Distributed Object Computing Conference (EDOC), 2007.

[5] J. P. Degabriele and D. Pym. Economic Aspects of a Utility Computing Service. Technical Report HPL-2007-101, HP Laboratories, 2007.

\footnotetext{
${ }^{1}$ http://www.google.com/advanced_search?hl=en

${ }^{2}$ http://search.yahoo.com/web/advanced?ei=UTF-8
}

[6] G. R. Gangadharan and V. D'Andrea. Licensing Services: Formal Analysis and Implementation. In Proceedings of the Fourth International Conference on Service Oriented Computing (ICSOC'06), Chicago, USA, pages 365-377, 2006.

[7] G. R. Gangadharan, V. D'Andrea, R. Iannella, and M. Weiss. ODRL Service Licensing Profile (ODRL-S). In Proceedings of the 5th International Workshop for Technical, Economic, and Legal Aspects of Business Models for Virtual Goods, 2007.

[8] S. Grimm, B. Motik, and C. Preist. Variance in e-Business Service Discovery. In Proceedings of the Semantic Web Services Workshop (in conjunction with ISWC 2004), 2004.

[9] O. Gunther, G. Tamm, and F. Leymann. Pricing Web Services. In Dagstuhl Seminar Proceedings 06291 (The Role of Business Processes in Service Oriented Architectures), 2006.

[10] R. Iannella, editor. Open Digital Rights Language (ODRL) Version 1.1, 2002. http://odrl.net/1.1/ODRL-11.pdf.

[11] Y. Liu, A. Ngu, and L. Zeng. QoS computation and policing in dynamic web service selection. In Proceedings of the 13th International World Wide Web Conference on Alternate Track Papers and Posters, 2004.

[12] H. Ludwig, A. Keller, A. Dan, R. King, and R. Franck. Web Service Level Agreement (WSLA) Language Specification. IBM Coporation, 2003.

[13] L. Rosen. Open Source Licensing: Software Freedom and Intellectual Property Law . Prentice Hall, 2004.

[14] J. Skene, D. Lamanna, and W. Emmerich. Precise Service Level Agreements. In Proceedings of 26th International Conference on Software Engineering (ICSE), 2004.

[15] V. Tosic, B. Pagurek, K. Patel, B. Esfandiari, and W. Ma. Management Applications of the Web Service Offerings Language. In Proceedings of the 15th Conference on Advanced Information Systems Engineering, 2003.

[16] A. Vedamuthu, D. Orchard, F. Hirsch, M. Hondo, P. Yendluri, T. Boubez, and U. Yalcinalp. Web Services Policy Framework, 2007. http://www.w3.org/TR/ws-policy.

[17] X. Wang, T. Vitvar, M. Kerrigan, and I. Toma. A QoS-aware Selection Model for Semantic Web Services. In Proceedings of the Fourth International Conference on Service Oriented Computing (ICSOC), 2006.

[18] World Intellectual Property Organization. Successful Technology Licensing. WIPO Publishers, Geneva, Switzerland, 2004. 